\title{
Difference equations related to majorization theorems via Montgomery identity and Green's functions with application to the Shannon entropy
}

\author{
Nouman Siddique ${ }^{1}$, Muhammad Imran ${ }^{1 *}$ (D), Khuram Ali Khan ${ }^{2}$ and Josip Pečarić ${ }^{3}$
}

\section{"Correspondence:}

drmimranchaudhry@gmail.com 1 Department of Mathematics, Government College University, 38000 Faisalabad, Pakistan

Full list of author information is available at the end of the article

\section{勿 Springer}

\begin{abstract}
In this paper we give generalized results of a majorization inequality by using extension of the Montgomery identity and newly defined Green's functions (Mehmood et al. in J. Inequal. Appl. 2017(1):108, 2017). We obtain a generalized majorization theorem for the class of $n$-convex functions. We use Csiszár $f$-divergence and generalized majorization-type inequalities to obtain new generalized results. We further discuss our obtained generalized results in terms of the Shannon entropy and the Kullback-Leibler distance.
\end{abstract}

Keywords: Majorization inequality; Fuchs's theorem; Montgomery identity; New Green's functions; Čebyšev functional; Csiszár $f$-divergence; Kullback-Leibler divergence; Shannon entropy

\section{Introduction}

The theory of majorization is perhaps most remarkable for its simplicity. It is a powerful, easy-to-use, and flexible mathematical tool which can be applicable to a wide number of fields. The key contributors in majorization are Dalton [14], Hardy et al. [16], Lorenz [30], Muirhead [36], and Schur [43]. Many important contributions were also made by other authors. Particularly, the comprehensive survey by Ando [8] gives alternative derivations, generalizations, and a different viewpoint. For an elementary discussion of majorization, see Marshall and Olkin's monograph [32].

In 2018, Latif et al. [29] studied generalized results related to the majorization inequality by using Taylor's polynomial in combination with newly introduced Green's functions. In the same year, Siddique et al. [44] gave generalized majorization results via Lidstone's polynomial and newly defined Green's functions. The theory of majorization is widely used in many fields of application. In [21], Khan et al. presented significant material on majorization along with its applications in the field of information theory.

In this paper, our main goal is to obtain generalized results about majorization via new Green's functions and an extension of the Montgomery identity. We further make con-

(c) The Author(s) 2020. This article is licensed under a Creative Commons Attribution 4.0 International License, which permits use, sharing, adaptation, distribution and reproduction in any medium or format, as long as you give appropriate credit to the original author(s) and the source, provide a link to the Creative Commons licence, and indicate if changes were made. The images or other third party material in this article are included in the article's Creative Commons licence, unless indicated otherwise in a credit line to the material. If material is not included in the article's Creative Commons licence and your intended use is not permitted by statutory regulation or exceeds the permitted use, you will need to obtain permission directly from the copyright holder. To view a copy of this licence, visit http://creativecommons.org/licenses/by/4.0/. 
nection of majorization with information theory and discuss our generalized majorization inequality in terms of divergences and entropies. The results we obtain in this paper are closely related to the contents given in [1-5]. Moreover, some related results with the present topic can also be found in [10, 11, 27, 41, 42].

The following definition of majorization is from [39, page 319].

Definition 1 Let $\mathbf{x}=\left(x_{1}, \ldots, x_{m}\right), \mathbf{y}=\left(y_{1}, \ldots, y_{m}\right)$ be two real $m$-tuples. Then we say that $\mathbf{x}$ majorizes $\mathbf{y}$ (denoted by $\mathbf{x} \succ \mathbf{y}$ ) if, for $\lambda=1,2, \ldots, m-1$,

$$
\sum_{i=1}^{\lambda} y_{[i]} \leq \sum_{i=1}^{\lambda} x_{[i]}
$$

holds and

$$
\sum_{i=1}^{m} x_{i}=\sum_{i=1}^{m} y_{i}
$$

where $x_{[i]}$ and $y_{[i]}$ denote their nonincreasing order.

Note that, in the definition of majorization, the original order of $x_{i} \mathrm{~s}$ and $y_{i}$ s plays no role because real $m$-tuples can always be reordered nonincreasingly.

The following theorem is famed in literature as classical majorization theorem and is given in [33, page 11] (see also [39, page 320]).

Theorem 1 Let $I=\left[\zeta_{1}, \zeta_{2}\right] \subset \mathbb{R}$ and $\mathbf{x}=\left(x_{1}, \ldots, x_{m}\right), \mathbf{y}=\left(y_{1}, \ldots, y_{m}\right) \in I^{m}$ be two nonincreasing $m$-tuples. Then $\mathbf{x}$ majorizes $\mathbf{y}$ if and only if the following inequality holds:

$$
\sum_{i=1}^{m} f\left(y_{i}\right) \leq \sum_{i=1}^{m} f\left(x_{i}\right)
$$

where $f:\left[\zeta_{1}, \zeta_{2}\right] \rightarrow \mathbb{R}$ is a continuous convex function.

A generalization of the aforementioned theorem is regarded as weighted majorization theorem and is proved by Fuchs in [15] (see also [39, page 323]).

Theorem 2 Let $I=\left[\zeta_{1}, \zeta_{2}\right] \subset \mathbb{R}$ and $\mathbf{x}=\left(x_{1}, \ldots, x_{m}\right), \mathbf{y}=\left(y_{1}, \ldots, y_{m}\right) \in I^{m}$ be two nonincreasing m-tuples. Let $\mathbf{p}=\left(p_{1}, \ldots, p_{m}\right) \in \mathbb{R}^{m}$ be such that

$$
\sum_{i=1}^{\lambda} p_{i} y_{i} \leq \sum_{i=1}^{\lambda} p_{i} x_{i} \quad \text { for } \lambda=1,2, \ldots, m-1
$$

and

$$
\sum_{i=1}^{m} p_{i} y_{i}=\sum_{i=1}^{m} p_{i} x_{i}
$$


Then the following inequality holds:

$$
\sum_{i=1}^{m} p_{i} f\left(y_{i}\right) \leq \sum_{i=1}^{m} p_{i} f\left(x_{i}\right)
$$

where $f:\left[\zeta_{1}, \zeta_{2}\right] \rightarrow \mathbb{R}$ is a continuous convex function.

The following theorem represents an integral form of Theorem 2 and is in fact a simple consequence of Theorem 1 given in [37] (see also [39, page 328]).

Theorem 3 Let $\phi, \psi:[a, b] \rightarrow\left[\zeta_{1}, \zeta_{2}\right]$ be two continuous nonincreasing functions and $p:$ $[a, b] \rightarrow \mathbb{R}$ be continuous. If

$$
\int_{a}^{\lambda} p(w) \psi(w) d w \leq \int_{a}^{\lambda} p(w) \phi(w) d w \quad \text { for every } \lambda \in[a, b],
$$

and

$$
\int_{a}^{b} p(w) \psi(w) d w=\int_{a}^{b} p(w) \phi(w) d w
$$

hold, then

$$
\int_{a}^{b} p(w) f(\psi(w)) d w \leq \int_{a}^{b} p(w) f(\phi(w)) d w
$$

where $f:\left[\zeta_{1}, \zeta_{2}\right] \rightarrow \mathbb{R}$ is a continuous convex function.

For other forms of an integral version and generalization of the majorization theorem, see [33, page 583], [9, 22, 24-26, 28, 31]. In this paper, we present our results for nonincreasing functions $\phi$ and $\psi$ which satisfy the conditions of Theorem 3, but those results hold too for nondecreasing $\phi$ and $\psi$ satisfying the following inequality:

$$
\int_{\lambda}^{b} p(w) \psi(w) d w \leq \int_{\lambda}^{b} p(w) \phi(w) d w, \quad \text { for every } \lambda \in[a, b],
$$

and condition (6). For instance, see example in [33, page 584].

Definition 2 Let $I=\left[\zeta_{1}, \zeta_{2}\right] \subset \mathbb{R}$ and $f:\left[\zeta_{1}, \zeta_{2}\right] \rightarrow \mathbb{R}$ be a function. Then $n$th order divided difference of $f$ at distinct points $x_{0}, \ldots, x_{n} \in\left[\zeta_{1}, \zeta_{2}\right]$ is defined recursively (see $[6,39]$ ) by

$$
f\left[x_{i}\right]=f\left(x_{i}\right), \quad(i=0,1, \ldots, n)
$$

and

$$
f\left[x_{0}, \ldots, x_{n}\right]=\frac{f\left[x_{1}, \ldots, x_{n}\right]-f\left[x_{0}, \ldots, x_{n-1}\right]}{x_{n}-x_{0}} .
$$

Note that $n$th order divided difference of a function $f$ does not depend on the order of points. 
We can extend this definition by considering the condition that some (or all) points coincide. Assuming that $f^{(k-1)}$ exists, we define

$$
f \underbrace{[x, \ldots, x]}_{k \text {-times }}=\frac{f^{(k-1)}(x)}{(k-1) !} \text {. }
$$

Popoviciu [40] initially discussed the notion of $n$-convexity. We follow the definition given by Karlin [20].

Definition 3 A function $f:\left[\zeta_{1}, \zeta_{2}\right] \rightarrow \mathbb{R}$ is $n$-convex, $n \geq 0$ if

$$
f\left[x_{0}, \ldots, x_{n}\right] \geq 0
$$

holds for all choices of $(n+1)$ distinct points $x_{0}, \ldots, x_{n} \in\left[\zeta_{1}, \zeta_{2}\right]$.

Aljinović et al. in [7] proved the following proposition which gives an extension of the Montgomery identity via Taylor's formula.

Proposition 1 Let $f: I \rightarrow \mathbb{R}$ be such that $f^{(n-1)}$ is absolutely continuous, where $n \in \mathbb{N}$ and $I \subset \mathbb{R}$ is an open interval. Then, for $\zeta_{1}, \zeta_{2} \in I$ with $\zeta_{1}<\zeta_{2}$, the following identity holds:

$$
\begin{aligned}
f(x)= & \frac{1}{\zeta_{2}-\zeta_{1}} \int_{\zeta_{1}}^{\zeta_{2}} f(s) d s+\sum_{k=0}^{n-2} \frac{\left(x-\zeta_{1}\right)^{k+2} f^{(k+1)}\left(\zeta_{1}\right)}{k !(k+2)\left(\zeta_{2}-\zeta_{1}\right)} \\
& -\sum_{k=0}^{n-2} \frac{\left(x-\zeta_{2}\right)^{k+2} f^{(k+1)}\left(\zeta_{2}\right)}{k !(k+2)\left(\zeta_{2}-\zeta_{1}\right)}+\frac{1}{(n-1) !} \int_{\zeta_{1}}^{\zeta_{2}} T_{n}(x, s) f^{(n)}(s) d s,
\end{aligned}
$$

where

$$
T_{n}(x, s)= \begin{cases}-\frac{(x-s)^{n}}{n\left(\zeta_{2}-\zeta_{1}\right)}+\frac{x-\zeta_{1}}{\zeta_{2} \zeta_{1}}(x-s)^{n-1}, & \zeta_{1} \leq s \leq x, \\ -\frac{(x-s)^{n}}{n\left(\zeta_{2}-\zeta_{1}\right)}+\frac{x-\zeta_{2}}{\zeta_{2}-\zeta_{1}}(x-s)^{n-1}, & x<s \leq \zeta_{2}\end{cases}
$$

As a special case, for $n=1$, the sum $\sum_{k=0}^{n-2} \cdots$ in (10) is empty, so (10) reduces to the following famous Montgomery identity (see [35]):

$$
f(x)=\frac{1}{\zeta_{2}-\zeta_{1}} \int_{\zeta_{1}}^{\zeta_{2}} f(s) d s+\int_{\zeta_{1}}^{\zeta_{2}} P(x, s) f^{\prime}(s) d s
$$

where $P(x, s)$ is the Peano kernel given by

$$
P(x, s)= \begin{cases}\frac{s-\zeta_{1}}{\zeta_{\zeta_{1}-\zeta_{1}}}, & \zeta_{1} \leq s \leq x \\ \frac{s-\zeta_{2}}{\zeta_{2}-\zeta_{1}}, & x<s \leq \zeta_{2}\end{cases}
$$

As stated in [34], the complete reference about Abel-Gontscharoff polynomial and a theorem for 'two-point right focal problem' is given in [6]. 
Remark 1 Abel-Gontscharoff polynomial as a special choice for 'two-point right focal' interpolating polynomial for $n=2$ is as follows:

$$
f(z)=f\left(\zeta_{1}\right)+\left(z-\zeta_{1}\right) f^{\prime}\left(\zeta_{2}\right)+\int_{\zeta_{1}}^{\zeta_{2}} G_{\Omega, 2}(z, w) f^{\prime \prime}(w) d w
$$

where $G_{\Omega, 2}(z, w):\left[\zeta_{1}, \zeta_{2}\right] \times\left[\zeta_{1}, \zeta_{2}\right] \rightarrow \mathbb{R}$ is Green's function for 'two-point right focal problem' given by

$$
G_{1}(z, w)=G_{\Omega, 2}(z, w)= \begin{cases}\left(\zeta_{1}-w\right), & \zeta_{1} \leq w \leq z \\ \left(\zeta_{1}-z\right), & z \leq w \leq \zeta_{2}\end{cases}
$$

Motivated by Abel-Gontscharoff Green's function for 'two-point right focal problem', Mehmood et al. (see [34]) presented some new types of Green's functions which are continuous as well as convex, as follows:

Let $\left[\zeta_{1}, \zeta_{2}\right] \subset \mathbb{R}$. Define new types of Green's functions $G_{d}:\left[\zeta_{1}, \zeta_{2}\right] \times\left[\zeta_{1}, \zeta_{2}\right] \rightarrow \mathbb{R}$, where $d=2,3,4$, as follows:

$$
\begin{aligned}
& G_{2}(z, w)= \begin{cases}\left(z-\zeta_{2}\right), & \zeta_{1} \leq w \leq z, \\
\left(w-\zeta_{2}\right), & z \leq w \leq \zeta_{2},\end{cases} \\
& G_{3}(z, w)= \begin{cases}\left(z-\zeta_{1}\right), & \zeta_{1} \leq w \leq z, \\
\left(w-\zeta_{1}\right), & z \leq w \leq \zeta_{2},\end{cases} \\
& G_{4}(z, w)= \begin{cases}\left(\zeta_{2}-w\right), & \zeta_{1} \leq w \leq z \\
\left(\zeta_{2}-z\right), & z \leq w \leq \zeta_{2}\end{cases}
\end{aligned}
$$

The following lemma, given by Mehmood et al. [34], will help us to obtain the new generalizations of majorization inequality.

Lemma 1 Let $f:\left[\zeta_{1}, \zeta_{2}\right] \rightarrow \mathbb{R}$ be such that $f \in C^{2}\left(\left[\zeta_{1}, \zeta_{2}\right]\right)$ and $G_{d},(d=1,2,3,4)$ be Green's functions given in (15)-(18) respectively. Then along with identity (14) the following identities hold:

$$
\begin{aligned}
& f(z)=f\left(\zeta_{2}\right)+\left(\zeta_{2}-z\right) f^{\prime}\left(\zeta_{1}\right)+\int_{\zeta_{1}}^{\zeta_{2}} G_{2}(z, w) f^{\prime \prime}(w) d w \\
& f(z)=f\left(\zeta_{2}\right)-\left(\zeta_{2}-\zeta_{1}\right) f^{\prime}\left(\zeta_{2}\right)+\left(z-\zeta_{1}\right) f^{\prime}\left(\zeta_{1}\right)+\int_{\zeta_{1}}^{\zeta_{2}} G_{3}(z, w) f^{\prime \prime}(w) d w \\
& f(z)=f\left(\zeta_{1}\right)+\left(\zeta_{2}-\zeta_{1}\right) f^{\prime}\left(\zeta_{1}\right)-\left(\zeta_{2}-z\right) f^{\prime}\left(\zeta_{2}\right)+\int_{\zeta_{1}}^{\zeta_{2}} G_{4}(z, w) f^{\prime \prime}(w) d w .
\end{aligned}
$$

We organize this paper in the following way:

In Sect. 2, we give generalized results of the majorization inequality and related bounds by using an extension of the Montgomery identity and new Green's functions. In Sect. 3, we use Csiszár $f$-divergence and generalized majorization-type inequalities to obtain new generalized results. We further discuss our obtained generalized results in terms of the Shannon entropy and the Kullback-Leibler distance. 


\section{Generalized majorized identities and related bounds via Montgomery identity and new Green's functions}

Before starting this section, we first define some notations which will be used throughout this article.

Majorization difference for a continuous convex function $f$ is denoted as follows:

$$
\mathbb{D}(\mathbf{x}, \mathbf{y}, \mathbf{p}, f(\cdot)):=\sum_{i=1}^{m} p_{i} f\left(x_{i}\right)-\sum_{i=1}^{m} p_{i} f\left(y_{i}\right)
$$

where $\mathbf{x}, \mathbf{y}$, and $\mathbf{p}$ are as defined in Theorem 2. Similarly, the integral majorization difference for a continuous convex function $f$ is denoted as follows:

$$
\widetilde{\mathbb{D}}(\phi, \psi, p, f(\cdot)):=\int_{a}^{b} p(w)(f(\phi(w))-f(\psi(w))) d w
$$

where $\phi, \psi$, and $p$ are as defined in Theorem 3 .

The following theorem gives two equivalent statements between the weighted majorization inequality for a continuous convex function and the inequality involving newly defined Green's functions.

Theorem 4 Let $I=\left[\zeta_{1}, \zeta_{2}\right] \subset \mathbb{R}$ and $\mathbf{x}=\left(x_{1}, \ldots, x_{m}\right), \mathbf{y}=\left(y_{1}, \ldots, y_{m}\right) \in I^{m}$ be two nonincreasing m-tuples. Let $\mathbf{p}=\left(p_{1}, \ldots, p_{m}\right) \in \mathbb{R}^{m}$ be such that it satisfies (3) and $G_{d}(d=1,2,3,4)$ be as defined in (15)-(18) respectively. Then the following two assertions are equivalent:

(i) If $:\left[\zeta_{1}, \zeta_{2}\right] \rightarrow \mathbb{R}$ is a continuous convex function, we have

$$
\mathbb{D}(\mathbf{x}, \mathbf{y}, \mathbf{p}, f(\cdot)) \geq 0
$$

(ii) For $s \in\left[\zeta_{1}, \zeta_{2}\right]$, the following inequality holds:

$$
\mathbb{D}\left(\mathbf{x}, \mathbf{y}, \mathbf{p}, G_{d}(\cdot, s)\right) \geq 0, \quad d=1,2,3,4 .
$$

Proof Let assertion (i) hold. Then $G_{d}(\cdot, s)\left(s \in\left[\zeta_{1}, \zeta_{2}\right]\right)$, being continuous and convex, for fixed $d=1,2,3,4$ satisfies inequality (24), i.e., inequality (25) holds.

On the other hand, let assertion (ii) hold and $f:\left[\zeta_{1}, \zeta_{2}\right] \rightarrow \mathbb{R}$ be a convex function such that $f \in C^{2}\left(\left[\zeta_{1}, \zeta_{2}\right]\right)$. Then we can write the function $f$ in the forms (14), (19), (20), and (21) for Green's functions $G_{d}, d=1,2,3,4$, respectively. Hence using (3) and performing simple calculations, for all $s \in\left[\zeta_{1}, \zeta_{2}\right]$, we have

$$
\mathbb{D}(\mathbf{x}, \mathbf{y}, \mathbf{p}, f(\cdot))=\int_{\zeta_{1}}^{\zeta_{2}} \mathbb{D}\left(\mathbf{x}, \mathbf{y}, \mathbf{p}, G_{d}(\cdot, s)\right) f^{\prime \prime}(s) d s, \quad d=1,2,3,4 .
$$

Since $f$ is convex, $f^{\prime \prime}(s) \geq 0$ for all $s \in\left[\zeta_{1}, \zeta_{2}\right]$. Also, inequality (25) holds, so from (26) we get inequality (24).

One must note that in this proof, the demand for the existence of the second derivative of $f$ is not necessary ([39], page 172). We can directly eliminate this condition because it is possible to approximate uniformly continuous convex functions by convex polynomials.

The following theorem gives weighted majorization difference by using extension of the Montgomery identity and newly defined Green's functions. 
Theorem 5 Let all the assumptions of Theorem 2 hold. Let $f: I \rightarrow \mathbb{R}$ be such that $f^{(n-1)}$ is absolutely continuous, where $n \in \mathbb{N}(n \geq 3)$ and $I \subset \mathbb{R}$ is an open interval. Then, for $\zeta_{1}, \zeta_{2} \in I$ with $\zeta_{1}<\zeta_{2}$ and for all $s \in\left[\zeta_{1}, \zeta_{2}\right]$, we have the following identities:

$$
\begin{aligned}
\mathbb{D}(\mathbf{x}, \mathbf{y}, \mathbf{p}, f(\cdot))= & \sum_{k=1}^{n-1} \frac{k}{(k-1) !} \int_{\zeta_{1}}^{\zeta_{2}} \mathbb{D}\left(\mathbf{x}, \mathbf{y}, \mathbf{p}, G_{d}(\cdot, s)\right) \\
& \times \frac{f^{(k)}\left(\zeta_{1}\right)\left(s-\zeta_{1}\right)^{k-1}-f^{(k)}\left(\zeta_{2}\right)\left(s-\zeta_{2}\right)^{k-1}}{\zeta_{2}-\zeta_{1}} d s \\
& +\frac{1}{(n-3) !} \int_{\zeta_{1}}^{\zeta_{2}} f^{(n)}(t)\left(\int_{\zeta_{1}}^{\zeta_{2}} \mathbb{D}\left(\mathbf{x}, \mathbf{y}, \mathbf{p}, G_{d}(\cdot, s)\right) \widehat{T}_{n-2}(s, t) d s\right) d t,
\end{aligned}
$$

where

$$
\widehat{T}_{n-2}(s, t)= \begin{cases}\frac{1}{\zeta_{2}-\zeta_{1}}\left[\frac{(s-t)^{n-2}}{n-2}+\left(s-\zeta_{1}\right)(s-t)^{n-3}\right], & \zeta_{1} \leq t \leq s, \\ \frac{1}{\zeta_{2}-\zeta_{1}}\left[\frac{(s-t)^{n-2}}{n-2}+\left(s-\zeta_{2}\right)(s-t)^{n-3}\right], & s<t \leq \zeta_{2},\end{cases}
$$

and $G_{d}(d=1,2,3,4)$ are Green's functions defined in (15)-(18) respectively. Moreover, we have

$$
\begin{aligned}
\mathbb{D}(\mathbf{x}, \mathbf{y}, \mathbf{p}, f(\cdot))= & \sum_{k=1}^{n-1} \frac{k-2}{(k-1) !} \int_{\zeta_{1}}^{\zeta_{2}} \mathbb{D}\left(\mathbf{x}, \mathbf{y}, \mathbf{p}, G_{d}(\cdot, s)\right) \\
& \times \frac{f^{(k)}\left(\zeta_{1}\right)\left(s-\zeta_{1}\right)^{k-1}-f^{(k)}\left(\zeta_{2}\right)\left(s-\zeta_{2}\right)^{k-1}}{\zeta_{2}-\zeta_{1}} d s \\
& +\frac{1}{(n-3) !} \int_{\zeta_{1}}^{\zeta_{2}} f^{(n)}(t)\left(\int_{\zeta_{1}}^{\zeta_{2}} \mathbb{D}\left(\mathbf{x}, \mathbf{y}, \mathbf{p}, G_{d}(\cdot, s)\right) T_{n-2}(s, t) d s\right) d t
\end{aligned}
$$

where $T_{n-2}$ is as defined in (11).

Proof Using identities (14), (19), (20), and (21), for fixed $d=1,2,3$, 4, into weighted majorization difference (22), we get

$$
\mathbb{D}(\mathbf{x}, \mathbf{y}, \mathbf{p}, f(\cdot))=\int_{\zeta_{1}}^{\zeta_{2}} \mathbb{D}\left(\mathbf{x}, \mathbf{y}, \mathbf{p}, G_{d}(\cdot, s)\right) f^{\prime \prime}(s) d s
$$

Now, using an extension of the Montgomery identity given in (10) for the function $f(s)$ and after differentiating it twice with respect to $s$, we get

$$
\begin{aligned}
f^{\prime \prime}(s)= & \sum_{k=1}^{n-1} \frac{k}{(k-1) !} \frac{f^{(k)}\left(\zeta_{1}\right)\left(s-\zeta_{1}\right)^{k-1}-f^{(k)}\left(\zeta_{2}\right)\left(s-\zeta_{2}\right)^{k-1}}{\zeta_{2}-\zeta_{1}} \\
& +\frac{1}{(n-3) !} \int_{\zeta_{1}}^{\zeta_{2}} \widehat{T}_{n-2}(s, t) f^{(n)}(t) d t
\end{aligned}
$$

Using (31) in (30), we have

$$
\mathbb{D}(\mathbf{x}, \mathbf{y}, \mathbf{p}, f(\cdot))=\sum_{k=1}^{n-1} \frac{k}{(k-1) !} \int_{\zeta_{1}}^{\zeta_{2}} \mathbb{D}\left(\mathbf{x}, \mathbf{y}, \mathbf{p}, G_{d}(\cdot, s)\right)
$$




$$
\begin{aligned}
& \times \frac{f^{(k)}\left(\zeta_{1}\right)\left(s-\zeta_{1}\right)^{k-1}-f^{(k)}\left(\zeta_{2}\right)\left(s-\zeta_{2}\right)^{k-1}}{\zeta_{2}-\zeta_{1}} d s \\
& +\frac{1}{(n-3) !} \int_{\zeta_{1}}^{\zeta_{2}} \mathbb{D}\left(\mathbf{x}, \mathbf{y}, \mathbf{p}, G_{d}(\cdot, s)\right)\left(\int_{\zeta_{1}}^{\zeta_{2}} \widehat{T}_{n-2}(s, t) f^{(n)}(t) d t\right) d s
\end{aligned}
$$

Applying Fubini's theorem in the last term of (32), we get (27).

Also, replacing $f$ by $f^{\prime \prime}$ and $n$ by $n-2(n \geq 3)$ in $(10)$ and then rearranging indices, we have

$$
\begin{aligned}
f^{\prime \prime}(s)= & \frac{f^{\prime}\left(\zeta_{2}\right)-f^{\prime}\left(\zeta_{1}\right)}{\zeta_{2}-\zeta_{1}}+\sum_{k=3}^{n-1} \frac{k-2}{(k-1) !} \frac{f^{(k)}\left(\zeta_{1}\right)\left(s-\zeta_{1}\right)^{k-1}-f^{(k)}\left(\zeta_{2}\right)\left(s-\zeta_{2}\right)^{k-1}}{\zeta_{2}-\zeta_{1}} \\
& +\frac{1}{(n-3) !} \int_{\zeta_{1}}^{\zeta_{2}} T_{n-2}(s, t) f^{(n)}(t) d t
\end{aligned}
$$

which can also be written as

$$
\begin{aligned}
f^{\prime \prime}(s)= & \sum_{k=1}^{n-1} \frac{k-2}{(k-1) !} \frac{f^{(k)}\left(\zeta_{1}\right)\left(s-\zeta_{1}\right)^{k-1}-f^{(k)}\left(\zeta_{2}\right)\left(s-\zeta_{2}\right)^{k-1}}{\zeta_{2}-\zeta_{1}} \\
& +\frac{1}{(n-3) !} \int_{\zeta_{1}}^{\zeta_{2}} T_{n-2}(s, t) f^{(n)}(t) d t .
\end{aligned}
$$

Using (34) in (30) and then applying Fubini's theorem, we obtain (29).

An integral version of Theorem 5 is as follows.

Theorem 6 Let all the assumptions of Theorem 3 hold. Let $f: I \rightarrow \mathbb{R}$ be such that $f^{(n-1)}$ is absolutely continuous, where $n \in \mathbb{N}(n \geq 3)$ and $I \subset \mathbb{R}$ is an open interval. Then, for $\zeta_{1}, \zeta_{2} \in I$ with $\zeta_{1}<\zeta_{2}$ and for all $s \in\left[\zeta_{1}, \zeta_{2}\right]$, we have the following identities:

$$
\begin{aligned}
\widetilde{\mathbb{D}}(\phi, \psi, p, f(\cdot))= & \sum_{k=1}^{n-1} \frac{k}{(k-1) !} \int_{\zeta_{1}}^{\zeta_{2}} \tilde{\mathbb{D}}\left(\phi, \psi, p, G_{d}(\cdot, s)\right) \\
& \times \frac{f^{(k)}\left(\zeta_{1}\right)\left(s-\zeta_{1}\right)^{k-1}-f^{(k)}\left(\zeta_{2}\right)\left(s-\zeta_{2}\right)^{k-1}}{\zeta_{2}-\zeta_{1}} d s \\
& +\frac{1}{(n-3) !} \int_{\zeta_{1}}^{\zeta_{2}} f^{(n)}(t)\left(\int_{\zeta_{1}}^{\zeta_{2}} \tilde{\mathbb{D}}\left(\phi, \psi, p, G_{d}(\cdot, s)\right) \widehat{T}_{n-2}(s, t) d s\right) d t
\end{aligned}
$$

where $\widehat{T}_{n}$ is as defined in (28)and $G_{d}(d=1,2,3,4)$ are the Green's functions defined in (15)-(18) respectively. Moreover,

$$
\begin{aligned}
\tilde{\mathbb{D}}(\phi, \psi, p, f(\cdot))= & \sum_{k=1}^{n-1} \frac{k-2}{(k-1) !} \int_{\zeta_{1}}^{\zeta_{2}} \tilde{\mathbb{D}}\left(\phi, \psi, p, G_{d}(\cdot, s)\right) \\
& \times \frac{f^{(k)}\left(\zeta_{1}\right)\left(s-\zeta_{1}\right)^{k-1}-f^{(k)}\left(\zeta_{2}\right)\left(s-\zeta_{2}\right)^{k-1}}{\zeta_{2}-\zeta_{1}} d s \\
& +\frac{1}{(n-3) !} \int_{\zeta_{1}}^{\zeta_{2}} f^{(n)}(t)\left(\int_{\zeta_{1}}^{\zeta_{2}} \tilde{\mathbb{D}}\left(\phi, \psi, p, G_{d}(\cdot, s)\right) T_{n-2}(s, t) d s\right) d t
\end{aligned}
$$

where $T_{n-2}$ is as defined in (11). 
Proof Using identities (14), (19), (20), and (21), for fixed $d=1,2,3,4$, into the integral weighted majorization difference (23) and following similar steps as in the proof of Theorem 5 , we get required results.

A refinement of the weighted majorization-type inequality is presented in the following theorem.

Theorem 7 Let all the assumptions of Theorem 5 hold. Let $f: I \rightarrow \mathbb{R}$ be an $n$-convex function. If, for $d=1,2,3,4$,

$$
\int_{\zeta_{1}}^{\zeta_{2}} \mathbb{D}\left(\mathbf{x}, \mathbf{y}, \mathbf{p}, G_{d}(\cdot, s)\right) \widehat{T}_{n-2}(s, t) d s \geq 0 \quad \text { for all } t \in\left[\zeta_{1}, \zeta_{2}\right]
$$

then

$$
\begin{aligned}
\mathbb{D}(\mathbf{x}, \mathbf{y}, \mathbf{p}, f(\cdot)) \geq & \sum_{k=1}^{n-1} \frac{k}{(k-1) !} \int_{\zeta_{1}}^{\zeta_{2}} \mathbb{D}\left(\mathbf{x}, \mathbf{y}, \mathbf{p}, G_{d}(\cdot, s)\right) \\
& \times \frac{f^{(k)}\left(\zeta_{1}\right)\left(s-\zeta_{1}\right)^{k-1}-f^{(k)}\left(\zeta_{2}\right)\left(s-\zeta_{2}\right)^{k-1}}{\zeta_{2}-\zeta_{1}} d s .
\end{aligned}
$$

Moreover, if

$$
\int_{\zeta_{1}}^{\zeta_{2}} \mathbb{D}\left(\mathbf{x}, \mathbf{y}, \mathbf{p}, G_{d}(\cdot, s)\right) T_{n-2}(s, t) d s \geq 0 \quad \text { for all } t \in\left[\zeta_{1}, \zeta_{2}\right]
$$

then

$$
\begin{aligned}
\mathbb{D}(\mathbf{x}, \mathbf{y}, \mathbf{p}, f(\cdot)) \geq & \sum_{k=1}^{n-1} \frac{k-2}{(k-1) !} \int_{\zeta_{1}}^{\zeta_{2}} \mathbb{D}\left(\mathbf{x}, \mathbf{y}, \mathbf{p}, G_{d}(\cdot, s)\right) \\
& \times \frac{f^{(k)}\left(\zeta_{1}\right)\left(s-\zeta_{1}\right)^{k-1}-f^{(k)}\left(\zeta_{2}\right)\left(s-\zeta_{2}\right)^{k-1}}{\zeta_{2}-\zeta_{1}} d s .
\end{aligned}
$$

If we reverse the sign of inequalities in (37) and (39), then inequalities (38) and (40) are also reversed.

Proof As $f$ is an $n$-convex function, it follows that $f^{(n)} \geq 0$ (see [39], page 19 and page 293). Using this fact and substituting (37) and (39) in (27) and (29), respectively, we get the desired results.

An integral version of Theorem 7 is as follows.

Theorem 8 Let all the assumptions of Theorem 6 hold. Let $f: I \rightarrow \mathbb{R}$ be an n-convex function. If, for $d=1,2,3,4$,

$$
\int_{\zeta_{1}}^{\zeta_{2}} \widetilde{\mathbb{D}}\left(\phi, \psi, p, G_{d}(\cdot, s)\right) \widehat{T}_{n-2}(s, t) d s \geq 0 \quad \text { for all } t \in\left[\zeta_{1}, \zeta_{2}\right]
$$


then

$$
\begin{aligned}
\widetilde{\mathbb{D}}(\phi, \psi, p, f(\cdot)) \geq & \sum_{k=1}^{n-1} \frac{k}{(k-1) !} \int_{\zeta_{1}}^{\zeta_{2}} \widetilde{\mathbb{D}}\left(\phi, \psi, p, G_{d}(\cdot, s)\right) \\
& \times \frac{f^{(k)}\left(\zeta_{1}\right)\left(s-\zeta_{1}\right)^{k-1}-f^{(k)}\left(\zeta_{2}\right)\left(s-\zeta_{2}\right)^{k-1}}{\zeta_{2}-\zeta_{1}} d s .
\end{aligned}
$$

Moreover, if

$$
\int_{\zeta_{1}}^{\zeta_{2}} \tilde{\mathbb{D}}\left(\phi, \psi, p, G_{d}(\cdot, s)\right) T_{n-2}(s, t) d s \geq 0 \quad \text { for all } t \in\left[\zeta_{1}, \zeta_{2}\right]
$$

then

$$
\begin{aligned}
\widetilde{\mathbb{D}}(\phi, \psi, p, f(\cdot)) \geq & \sum_{k=1}^{n-1} \frac{k-2}{(k-1) !} \int_{\zeta_{1}}^{\zeta_{2}} \widetilde{\mathbb{D}}\left(\phi, \psi, p, G_{d}(\cdot, s)\right) \\
& \times \frac{f^{(k)}\left(\zeta_{1}\right)\left(s-\zeta_{1}\right)^{k-1}-f^{(k)}\left(\zeta_{2}\right)\left(s-\zeta_{2}\right)^{k-1}}{\zeta_{2}-\zeta_{1}} d s .
\end{aligned}
$$

If we reverse the sign of inequalities in (41) and (43), then inequalities (42) and (44) are also reversed.

Proof Using (41) and (43) in (35) and (36) respectively and following similar steps as in the proof of Theorem 7 , we get required results.

Theorem 9 Let all the assumptions of Theorem 5 be true. Iff is $n$-convex, where $n$ is even, then inequalities (38) and (40) hold.

Proof Since $G_{d}$ is continuous as well as convex for $d=1,2,3,4$, therefore from Theorem 2 we can write

$$
\mathbb{D}\left(\mathbf{x}, \mathbf{y}, \mathbf{p}, G_{d}(\cdot, s)\right) \geq 0
$$

Note that, when $n-2$ is even, $\widehat{T}_{n-2}(s, t)$ and $T_{n-2}(s, t)$ are nonnegative, so (37) and (39) hold. Now, using Theorem 7 , we get the required results.

An integral version of Theorem 9 is as follows.

Theorem 10 Let all the assumptions of Theorem 6 be true. Iff is $n$-convex, where $n$ is even, then inequalities (42) and (44) hold.

Proof Similar to the proof of Theorem 9.

The following corollary gives a generalized majorization theorem, i.e., Fuchs's theorem for $n$-convex functions. 
Corollary 1 Let all the assumptions of Theorem 9 be true. If the functions $\mathcal{F}_{1}, \mathcal{F}_{2}$ : $\left[\zeta_{1}, \zeta_{2}\right] \rightarrow \mathbb{R}$, given by

$$
\mathcal{F}_{1}(\cdot)=\sum_{k=1}^{n-1} \frac{k}{(k-1) !} \int_{\zeta_{1}}^{\zeta_{2}} G_{d}(\cdot, s) \frac{f^{(k)}\left(\zeta_{1}\right)\left(s-\zeta_{1}\right)^{k-1}-f^{(k)}\left(\zeta_{2}\right)\left(s-\zeta_{2}\right)^{k-1}}{\zeta_{2}-\zeta_{1}} d s
$$

and

$$
\mathcal{F}_{2}(\cdot)=\sum_{k=1}^{n-1} \frac{k-2}{(k-1) !} \int_{\zeta_{1}}^{\zeta_{2}} G_{d}(\cdot, s) \frac{f^{(k)}\left(\zeta_{1}\right)\left(s-\zeta_{1}\right)^{k-1}-f^{(k)}\left(\zeta_{2}\right)\left(s-\zeta_{2}\right)^{k-1}}{\zeta_{2}-\zeta_{1}} d s,
$$

are convex, then the right-hand sides of (42) and (44) are nonnegative, i.e., (4) is satisfied.

Proof Note that inequalities (38) and (40) can be written as follows:

$$
\mathbb{D}(\mathbf{x}, \mathbf{y}, \mathbf{p}, f(\cdot)) \geq \mathbb{D}\left(\mathbf{x}, \mathbf{y}, \mathbf{p}, \mathcal{F}_{i}(\cdot)\right), \quad i=1,2 .
$$

Now, the use of convex functions $\mathcal{F}_{i}, i=1,2$, in (4) lead us to the nonnegativity of the right-hand side of (48), which gives the required result.

Remark 2 As given for previous theorems, we can obtain an integral version of Corollary 1 , which is a generalization of the integral majorization theorem.

\section{Remarks 1}

(i) We can obtain upper bounds like Grüss- and Ostrowski-type inequalities for our obtained generalized identities. We can also present Lagrange and Cauchy-type mean value theorems by using linear functionals deduced from our generalized results (see for example $[29,38,44]$ ).

(ii) We can use an elegant method introduced by Jakšetić and Pečarć $[18,19]$ (see also $[23,34])$ to give $n$-exponential convexity, exponential convexity, and log-convexity, with the help of linear functionals deduced from our generalized results, on a given family with the same property for both discrete and integral cases. For more details, see [38].

\section{Csiszár f-divergence for majorization}

This section belongs to the study of generalized majorization-type inequality (38) in the form of divergences and entropies. We use Csiszár $f$-divergence and generalized majorization-type inequalities to obtain new generalized results. Moreover, results related to the Shannon entropy and the Kullback-Leibler (K-L) distance are also discussed.

The following notion of $f$-divergence was introduced by Csiszár in [12]. For more details, see [13].

Definition 4 Let $f: \mathbb{R}_{+} \rightarrow \mathbb{R}_{+}$be a convex function. If $\mathbf{r}=\left(r_{1}, \ldots, r_{m}\right)$ and $\mathbf{w}=\left(w_{1}, \ldots, w_{m}\right)$ are two positive probability distributions, then the $f$-divergence functional is

$$
I_{f}(\mathbf{r}, \mathbf{w}):=\sum_{i=1}^{m} w_{i} f\left(\frac{r_{i}}{w_{i}}\right)
$$


Note that in the $f$-divergence functional, nonnegative probability distributions can also be used by defining

$$
f(0):=\lim _{t \rightarrow 0^{+}} f(t) ; \quad \text { of }\left(\frac{0}{0}\right):=0 ; \quad 0 f\left(\frac{a}{t}\right):=0, \quad a>0 .
$$

In [17], Horváth et al. considered the following functionality based on the previous definition.

Definition 5 Let $J \subset \mathbb{R}$ be an interval and $f: J \rightarrow \mathbb{R}$ be an $n$-convex function. Let $\mathbf{r}=$ $\left(r_{1}, \ldots, r_{m}\right) \in \mathbb{R}^{m}$ and $\mathbf{w}=\left(w_{1}, \ldots, w_{m}\right) \in \mathbb{R}_{+}^{m}$ such that $\frac{r_{i}}{w_{i}} \in J, i=1,2, \ldots, m$. Then

$$
\widetilde{I}_{f}(\mathbf{r}, \mathbf{w}):=\sum_{i=1}^{m} w_{i} f\left(\frac{r_{i}}{w_{i}}\right) .
$$

Let $\mathbf{r}=\left(r_{1}, \ldots, r_{m}\right)$ and $\mathbf{w}=\left(w_{1}, \ldots, w_{m}\right)$ be two $\mathrm{m}$-tuples. Onwards now, we use the following notations in this article, i.e.,

$$
\frac{\mathbf{r}}{\mathbf{w}}:=\left(\frac{r_{1}}{w_{1}}, \frac{r_{2}}{w_{2}}, \ldots, \frac{r_{m}}{w_{m}}\right) \quad \text { and } \quad \widetilde{I}_{G_{d}}(\mathbf{r}, \mathbf{w}, s):=\sum_{i=1}^{m} w_{i} G_{d}\left(\frac{r_{i}}{w_{i}}, s\right)
$$

The following theorem connects the generalized majorization-type inequality given in Theorem 9 and Csiszár $f$-divergence.

Theorem 11 Let $f: I \rightarrow \mathbb{R}$ be such that $f^{(n-1)}$ is absolutely continuous, where $n \in \mathbb{N}(n>3)$ and $I \subset \mathbb{R}$ is an open interval. Let $G_{d}(d=1,2,3,4)$ be as defined in (15)-(18) respectively. Also, let $\mathbf{q}=\left(q_{1}, \ldots, q_{m}\right), \mathbf{r}=\left(r_{1}, \ldots, r_{m}\right) \in \mathbb{R}^{m}$, and $\mathbf{w}=\left(w_{1}, \ldots, w_{m}\right) \in \mathbb{R}_{+}^{m}$. Let

$$
\sum_{i=1}^{\lambda} r_{i} \leq \sum_{i=1}^{\lambda} q_{i}
$$

for $\lambda=1,2, \ldots, m-1$ and

$$
\sum_{i=1}^{m} r_{i}=\sum_{i=1}^{m} q_{i}
$$

with $\frac{q_{i}}{w_{i}}, \frac{r_{i}}{w_{i}} \in I(i=1,2, \ldots, m)$. If $\frac{\mathbf{q}}{\mathbf{w}}$ and $\frac{\mathbf{r}}{\mathbf{w}}$ are decreasing and $f$ is an $n$-convex function for $n=$ even $(n>3)$, then

$$
\begin{aligned}
\widetilde{I}_{f}(\mathbf{q}, \mathbf{w}) \geq & \widetilde{I}_{f}(\mathbf{r}, \mathbf{w})+\sum_{k=1}^{n-1} \frac{k}{(k-1) !} \int_{\zeta_{1}}^{\zeta_{2}}\left(\widetilde{I}_{G_{d}}(\mathbf{q}, \mathbf{w}, s)-\widetilde{I}_{G_{d}}(\mathbf{r}, \mathbf{w}, s)\right) \\
& \times \frac{f^{(k)}\left(\zeta_{1}\right)\left(s-\zeta_{1}\right)^{k-1}-f^{(k)}\left(\zeta_{2}\right)\left(s-\zeta_{2}\right)^{k-1}}{\zeta_{2}-\zeta_{1}} d s .
\end{aligned}
$$

Proof Take $x_{i}=\frac{q_{i}}{w_{i}}, y_{i}=\frac{r_{i}}{w_{i}}$, and $p_{i}=w_{i}>0(i=1,2, \ldots, m)$, then conditions (49) and (50) imply that conditions (2) and (3) hold. So, using these substitutions in (38), we get (51). 
Theorem 12 Let $g: I \rightarrow \mathbb{R}$ be a function. If, for $f(x):=x g(x), x \in I$, all the conditions of Theorem 11 hold, then

$$
\begin{aligned}
\widehat{I}_{g}(\mathbf{q}, \mathbf{w}):= & \sum_{i=1}^{m} q_{i} g\left(\frac{q_{i}}{w_{i}}\right) \\
\geq & \left.\widehat{I}_{g}(\mathbf{r}, \mathbf{w})+\sum_{k=1}^{n-1} \frac{k}{(k-1) !} \int_{\zeta_{1}}^{\zeta_{2}} \widetilde{I}_{G_{d}}(\mathbf{q}, \mathbf{w}, s)-\widetilde{I}_{G_{d}}(\mathbf{r}, \mathbf{w}, s)\right) \\
& \times \frac{(x g)^{(k)}\left(\zeta_{1}\right)\left(s-\zeta_{1}\right)^{k-1}-(x g)^{(k)}\left(\zeta_{2}\right)\left(s-\zeta_{2}\right)^{k-1}}{\zeta_{2}-\zeta_{1}} d s .
\end{aligned}
$$

Proof Following the proof of Theorem 11 for $f(x):=x g(x)$, we get (52).

The notion of entropic measure of disorder and the theory of majorization are closely related. Next we present two special cases for majorization relations with the connection to entropic inequalities.

In the first case we discuss a generalized majorization-type inequality with the entropy of a discrete probability distribution.

Definition 6 Let $\mathbf{r}=\left(r_{1}, \ldots, r_{m}\right)$ be a positive probability distribution. Then the Shannon entropy of $\mathbf{r}$ is defined as follows:

$$
S(\mathbf{r}):=-\sum_{i=1}^{m} r_{i} \log r_{i} .
$$

Note that the definition does not provide any problem for the zero probability case, because $\lim _{x \rightarrow 0} x \log x=0$.

Corollary 2 Let $\mathbf{q}=\left(q_{1}, \ldots, q_{m}\right), \mathbf{r}=\left(r_{1}, \ldots, r_{m}\right) \in \mathbb{R}_{+}^{m}$, and $\mathbf{w}=\left(w_{1}, \ldots, w_{m}\right)$ be a positive probability distribution such that conditions (49) and (50) hold with $\frac{q_{i}}{w_{i}}, \frac{r_{i}}{w_{i}} \in I(i=$ $1,2, \ldots, m)$. If $\log$ has base $b$ greater than 1 and $\frac{\mathbf{q}}{\mathbf{w}}$ and $\frac{\mathbf{r}}{\mathbf{w}}$ are decreasing, then for the Shannon entropy of $\mathbf{w}$, the following estimate holds:

$$
\begin{aligned}
S(\mathbf{w}) \leq & \left.\sum_{i=1}^{m} w_{i} \log \left(\frac{r_{i}}{w_{i}}\right)-\sum_{k=1}^{n-1} \frac{k}{(k-1) !} \int_{\zeta_{1}}^{\zeta_{2}} \widetilde{I}_{G_{d}}(\mathbf{q}, \mathbf{w}, s)-\widetilde{I}_{G_{d}}(\mathbf{r}, \mathbf{w}, s)\right) \\
& \times \frac{1}{\zeta_{2}-\zeta_{1}}\left(\frac{(-1)^{k}(k-1) !}{\zeta_{1}^{k} \ln b}\left(s-\zeta_{1}\right)^{k-1}-\frac{(-1)^{k}(k-1) !}{\zeta_{2}^{k} \ln b}\left(s-\zeta_{2}\right)^{k-1}\right) d s .
\end{aligned}
$$

If $\log$ has base b between 0 and 1 , then inequality (53) is reversed.

Proof Take $f(x):=-\log x$, which is an $n$-convex function for $n=$ even $(n>3)$ and $q_{i}=1$ $(i=1,2, \ldots, m)$. Then, by using Theorem 11, we get (53). Moreover, for $n=$ odd $(n>3)$, the inequality in (53) is reversed.

Corollary 3 Let $\mathbf{q}=\left(q_{1}, \ldots, q_{m}\right)$ and $\mathbf{r}=\left(r_{1}, \ldots, r_{m}\right)$ be two positive probability distributions such that conditions (49) and (50) hold with $q_{i}, r_{i} \in I(i=1,2, \ldots, m)$. If $\log$ has base $b$ 
greater than 1 and $\mathbf{q}$ and $\mathbf{r}$ are decreasing, then the relation between the Shannon entropies of $\mathbf{q}$ and $\mathbf{r}$ is given by the following estimate:

$$
\begin{aligned}
S(\mathbf{q}) \leq & S(\mathbf{r})-\sum_{k=1}^{n-1} \frac{k}{(k-1) !} \int_{\zeta_{1}}^{\zeta_{2}}\left(\widetilde{I}_{G_{d}}(\mathbf{q}, \mathbf{w}, s)-\widetilde{I}_{G_{d}}(\mathbf{r}, \mathbf{w}, s)\right) \\
& \times \frac{(x \log x)^{(k)}\left(\zeta_{1}\right)\left(s-\zeta_{1}\right)^{k-1}-(x \log x)^{(k)}\left(\zeta_{2}\right)\left(s-\zeta_{2}\right)^{k-1}}{\zeta_{2}-\zeta_{1}} d s,
\end{aligned}
$$

where for $u=1,2,(x \log x)^{\prime}\left(\zeta_{u}\right)=\frac{1}{\ln b}\left(1+\ln \zeta_{u}\right)$ and $(x \log x)^{(k)}\left(\zeta_{u}\right)=\frac{(-1)^{k}(k-2) !}{\zeta_{u}^{k-1} \ln b}, k \geq 2$. If $\log$ has base $b$ between 0 and 1 , then inequality (54) is reversed.

Proof Take $g(x):=\log x$ so that $x g(x):=x \log x$ is an $n$-convex function for $n=$ even $(n>3)$ and $w_{i}=1(i=1,2, \ldots, m)$. Then, by using Theorem 12, we get (54). Moreover, for $n=$ odd $(n>3)$, the inequality in (54) is reversed.

In the second case we study a generalized majorization-type inequality in terms of the $\mathrm{K}-\mathrm{L}$ distance or relative entropy between two probability distributions.

Definition 7 Let $\mathbf{r}=\left(r_{1}, \ldots, r_{m}\right)$ and $\mathbf{w}=\left(w_{1}, \ldots, w_{m}\right)$ be two positive probability distributions. Then the $\mathrm{K}-\mathrm{L}$ distance between them is defined by

$$
L(\mathbf{r}, \mathbf{w}):=\sum_{i=1}^{m} r_{i} \log \left(\frac{r_{i}}{w_{i}}\right)
$$

Corollary 4 Let $\mathbf{q}=\left(q_{1}, \ldots, q_{m}\right), \mathbf{r}=\left(r_{1}, \ldots, r_{m}\right), \mathbf{w}=\left(w_{1}, \ldots, w_{m}\right) \in \mathbb{R}_{+}^{m}$ such that conditions (49) and (50) hold with $\frac{q_{i}}{w_{i}}, \frac{r_{i}}{w_{i}} \in I(i=1,2, \ldots, m)$. If $\log$ has base $b$ greater than 1 and $\frac{\mathbf{q}}{\mathbf{w}}$ and $\frac{\mathbf{r}}{\mathbf{w}}$ are decreasing, then

$$
\begin{aligned}
& \sum_{i=1}^{m} w_{i} \log \left(\frac{q_{i}}{w_{i}}\right) \\
& \leq \sum_{i=1}^{m} w_{i} \log \left(\frac{r_{i}}{w_{i}}\right)-\sum_{k=1}^{n-1} \frac{k}{(k-1) !} \int_{\zeta_{1}}^{\zeta_{2}}\left(\widetilde{I}_{G_{d}}(\mathbf{q}, \mathbf{w}, s)-\widetilde{I}_{G_{d}}(\mathbf{r}, \mathbf{w}, s)\right) \\
& \quad \times \frac{1}{\zeta_{2}-\zeta_{1}}\left(\frac{(-1)^{k}(k-1) !}{\zeta_{1}^{k} \ln b}\left(s-\zeta_{1}\right)^{k-1}-\frac{(-1)^{k}(k-1) !}{\zeta_{2}^{k} \ln b}\left(s-\zeta_{2}\right)^{k-1}\right) d s
\end{aligned}
$$

If $\log$ has base b between 0 and 1 , then inequality (55) is reversed.

Proof Take $f(x):=-\log x$, which is an $n$-convex function for $n=$ even $(n>3)$. Then, by Theorem 11, we get (55). Moreover, for $n=\operatorname{odd}(n>3)$, the inequality in (55) is reversed.

Corollary 5 Let $\mathbf{q}=\left(q_{1}, \ldots, q_{m}\right), \mathbf{r}=\left(r_{1}, \ldots, r_{m}\right)$, and $\mathbf{w}=\left(w_{1}, \ldots, w_{m}\right)$ be positive probability distributions such that conditions (49) and (50) hold with $\frac{q_{i}}{w_{i}}, \frac{r_{i}}{w_{i}} \in I(i=1,2, \ldots, m)$. If $\log$ has base $b$ greater than 1 and $\frac{\mathrm{q}}{\mathrm{w}}$ and $\frac{\mathbf{r}}{\mathrm{w}}$ are decreasing, then the relation between the 
$K-L$ distance of $(\mathbf{r}, \mathbf{w})$ and $(\mathbf{q}, \mathbf{w})$ is given by the following estimate:

$$
\begin{aligned}
L(\mathbf{q}, \mathbf{w}) \geq & \left.L(\mathbf{r}, \mathbf{w})+\sum_{k=1}^{n-1} \frac{k}{(k-1) !} \int_{\zeta_{1}}^{\zeta_{2}} \widetilde{I}_{G_{d}}(\mathbf{q}, \mathbf{w}, s)-\widetilde{I}_{G_{d}}(\mathbf{r}, \mathbf{w}, s)\right) \\
& \times \frac{(x \log x)^{(k)}\left(\zeta_{1}\right)\left(s-\zeta_{1}\right)^{k-1}-(x \log x)^{(k)}\left(\zeta_{2}\right)\left(s-\zeta_{2}\right)^{k-1}}{\zeta_{2}-\zeta_{1}} d s,
\end{aligned}
$$

where for $u=1,2,(x \log x)^{\prime}\left(\zeta_{u}\right)=\frac{1}{\ln b}\left(1+\ln \zeta_{u}\right)$ and $(x \log x)^{(k)}\left(\zeta_{u}\right)=\frac{(-1)^{k}(k-2) !}{\zeta_{u}^{k-1} \ln b}, k \geq 2$. If $\log$ has base $b$ between 0 and 1 , then inequality (56) is reversed.

Proof Take $g(x):=\log x$ so that $x g(x):=x \log x$ is an $n$-convex function for $n=$ even $(n>3)$. Then, by using Theorem 12, we get (56). Moreover, for $n=$ odd $(n>3)$, the inequality in (56) is reversed.

Remark 3 In Sect. 3, we use generalized majorization-type inequality (38) to obtain results in terms of the Shannon entropy and the K-L distance. Following the same way, we can also give all these results related to the Shannon entropy and the K-L distance by using the generalized majorization-type inequality given in (40).

\section{Acknowledgements}

The authors are thankful to the anonymous referees for reading the manuscript and for giving fruitful comments and suggestions. The Ministry of Education and Science of Russian Federation has supported the research of the 4th author (Agreement No. 02.a03.21.0008).

\section{Funding}

There is no funding for this work.

\section{Availability of data and materials}

Data sharing not applicable to this article as no datasets were generated or analysed during the current study.

\section{Competing interests}

The authors declare that there is no conflict of interests regarding the publication of this paper.

\section{Authors' contributions}

All the authors have contributed equally in this research. All authors read and approved the final manuscript.

\section{Author details}

'Department of Mathematics, Government College University, 38000 Faisalabad, Pakistan. ${ }^{2}$ Department of Mathematics, University of Sargodha, 40100 Sargodha, Pakistan. ${ }^{3}$ Rudn University, Moscow, Russia.

\section{Publisher's Note}

Springer Nature remains neutral with regard to jurisdictional claims in published maps and institutional affiliations.

Received: 26 March 2020 Accepted: 6 August 2020 Published online: 18 August 2020

\section{References}

1. Adeel, M., Khan, K.A., Pečarić, Đ., Pečarić, J.: Generalization of the Levinson inequality with applications to information theory. J. Inequal. Appl. 2019, 230 (2019)

2. Adeel, M., Khan, K.A., Pečarić, Đ., Pečarić, J.: Levinson type inequalities for higher order convex functions via Abel-Gontscharoff interpolation. Adv. Differ. Equ. 2019, 430 (2019)

3. Adeel, M., Khan, K.A., Pečarić, Đ., Pečarić, J.: Estimation of $f$-divergence and Shannon entropy by Levinson type inequalities via new Green's functions and Lidstone polynomial. Adv. Differ. Equ. 2020, 27 (2020)

4. Adeel, M., Khan, K.A., Pečarić, Đ., Pečarić, J.: Estimation of $f$-divergence and Shannon entropy by using Levinson type inequalities for higher order convex functions via Hermite interpolating polynomial. J. Inequal. Appl. 2020, 137 (2020)

5. Adeel, M., Khan, K.A., Pečarić, Đ., Pečarić, J.: Estimation of $f$-divergence and Shannon entropy by Levinson type inequalities for higher order convex functions via Taylor polynomial. J. Math. Comput. Sci. 21(4), 322-334 (2020)

6. Agarwal, R.P., Wong, P.J.Y.: Error Inequalities in Polynomial Interpolation and Their Applications. Kluwer Academic, Dordrecht (1993)

7. Aljinović, A.A., Pečarić, J., Vukelić, A.: On some Ostrowski type inequalities via Montgomery identity and Taylor's formula II. Tamkang J. Math. 36(4), 279-301 (2005) 
8. Ando, T.: Majorization, doubly stochastic matrices, and comparison of eigenvalues. Linear Algebra Appl. 118, 163-248 (1989)

9. Bernett, N.S., Cerone, P., Dragomir, S.S.: Majorization inequalities for Stieltjes integrals. Appl. Math. Lett. 22, 416-421 (2009)

10. Cicalese, F., Gargano, L., Vaccaro, U.: Bounds on the entropy of a function of a random variable and their applications. IEEE Trans. Inf. Theory 64(4), 2220-2230 (2018)

11. Cicalese, F., Gargano, L., Vaccaro, U.: Minimum-entropy couplings and their applications. IEEE Trans. Inf. Theory 65(6), 3436-3451 (2019)

12. Csiszár, l.: Information-type measures of differences of probability distributions and indirect observations. Studia Sci. Math. Hung. 2, 299-318 (1967)

13. Csiszár, I.: Information measures: a critical survey. In: Trans. 7th Prague Conf. on Info. Th., Statist. Decis. Funct., Random Processes and 8th European Meeting of Statist., vol. B, pp. 73-86. Academia, Prague (1978)

14. Dalton, H.: The measurement of the inequality of incomes. Econ. J. 30(119), 348-361 (1920)

15. Fuchs, L.: A new proof of an inequality of Hardy-Littlewood-Pólya. Mat. Tidsskr., B 53-54 (1947)

16. Hardy, G.H., Littlewood, J.E., Pólya, G.: Some simple inequalities satisfied by convex functions. Messenger Math. 58 145-152 (1929)

17. Horváth, L., Pečarić, Đ., Pečarić, J.: Estimations of $f$ - and Rényi divergences by using a cyclic refinement of the Jensen's inequality. Bull. Malays. Math. Sci. Soc. 42(1), 933-946 (2017)

18. Jakšetić, J., Pečarić, J.: Exponential convexity method. J. Convex Anal. 20(1), 181-197 (2013)

19. Jakšetić, J., Pečarić, J., Perušić, A.: Steffensen inequality, higher order convexity and exponential convexity. Rend. Circ Mat. Palermo 63(1), 109-127 (2014)

20. Karlin, S.: Total Positivity. Stanford University Press, Stanford (1968)

21. Khan, M.A., Bradanović, S.I., Latif, N., Pečarić, Đ., Pečarić, J.: Majorization Inequality and Information Theory. Element, Zagreb (2019)

22. Khan, M.A., Khalid, S., Pečarić, J.: Refinements of some majorization type inequalities. J. Math. Inequal. 7(1), 73-92 (2013)

23. Khan, M.A., Latif, N., Pečarić, J.: Generalization of majorization theorem. J. Math. Inequal. 9(3), 847-872 (2015)

24. Khan, M.A., Latif, N., Perić, I., Pečarić, J.: On Sapogov's extension of Čebyšev's inequality. Thai J. Math. 10(2), 617-633 (2012)

25. Khan, M.A., Latif, N., Perić, I., Pečarić, J.: On majorization for matrices. Math. Balk. 27(1-2), 3-19 (2013)

26. Khan, M.A., Niezgoda, M., Pečarić, J.: On a refinement of the majorization type inequality. Demonstr. Math. 44(1), 49-57 (2011)

27. Latif, N., Pečarić, Đ., Pečarić, J.: Majorization, Csiszár divergence and Zipf-Mandelbrot law. J. Inequal. Appl. 2017(1), 1 (2017)

28. Latif, N., Pečarić, J., Perić, I.: On majorization, Favard's and Berwald's inequalities. Ann. Funct. Anal. 2(1), 31-50 (2011)

29. Latif, N., Siddique, N., Pečarić, J.: Generalization of majorization theorem-II. J. Math. Inequal. 12(3), 731-752 (2018)

30. Lorenz, M.O.: Methods of measuring concentration of wealth. Publ. Am. Stat. Assoc. 9(70), 209-219 (1905)

31. Maligranda, L., Pečarić, J., Persson, L.E.: Weighted Favard's and Berwald's inequalities. J. Math. Anal. Appl. 190, 248-262 (1995)

32. Marshall, A.W., Olkin, I.: Inequalities via majorization—an introduction. In: General Inequalities 3, pp. 165-187. Birkhäuser, Basel (1983)

33. Marshall, A.W., Olkin, I., Arnold, B.C.: Inequalities: Theory of Majorization and Its Applications, 2nd edn. Springer Series in Statistics. Springer, New York (2011)

34. Mehmood, N., Agarwal, R.P., Butt, S.I., Pečarić, J.: New generalization of Popoviciu type inequalities via new Green functions and Montgomery identity. J. Inequal. Appl. 2017(1), 108 (2017)

35. Mitrinović, D.S., Pečarić, J.E., Fink, A.M.: Inequalities for Functions and Their Integrals and Derivatives. Kluwer Academic, Dordrecht (1994)

36. Muirhead, R.F.: Some methods applicable to identities and inequalities of symmetric algebraic functions of $n$ letters Proc. Edinb. Math. Soc. 21, 144-157 (1903)

37. Pečarić, J.: On some inequalities for functions with nondecreasing increments. J. Math. Anal. Appl. 98, 188-197 (1984)

38. Pečarić, J., Perić, J.: Improvement of the Giaccardi and the Petrović inequality and related Stolarsky type means. An. Univ. Craiova, Ser. Mat. Inform. 39(1), 65-75 (2012)

39. Pečarić, J., Proschan, F., Tong, Y.L.: Convex Functions, Partial Orderings and Statistical Applications. Academic Press, New York (1992)

40. Popoviciu, T.: Sur l'approximation des fonctions convexes d'ordre superier. Mathematica 10,49-54 (1934)

41. Sason, l.: Tight bounds on the Rényi entropy via majorization with applications to guessing and compression. Entropy 20(12), 896 (2018)

42. Sason, I.: On data-processing and majorization inequalities for $f$-divergences with applications. Entropy $21(10), 1022$ (2019)

43. Schur, I.: Über eine Klasse von Mittelbildungen mit Anwendungen die Determinanten-Theorie. Sitzungsber. Berl. Math. Ges. 22, 9-20 (1923)

44. Siddique, N., Latif, N., Pečarić, J.: Generalized results of majorization inequality via Lidstone's polynomial and newly Green functions. J. Nonlinear Sci. Appl. 11(6), 812-831 (2018) 